\title{
Options thérapeutiques chez un patient diabétique de type 2 mal contrôlé par une insuline basale
}

\author{
Prs ANDRÉ J. SCHEEN ${ }^{\mathrm{a}, \mathrm{b}}$ et NICOLAS PAQUOT ${ }^{\mathrm{a}}$ \\ Rev Med Suisse 2017; 13: 1416-20
}

\begin{abstract}
Chez un patient diabétique de type 2 imparfaitement contrôlé sous une combinaison insuline basale-metformine, plusieurs options thérapeutiques peuvent être envisagées: une intensification de l'insulinothérapie avec différents schémas (titration avec un nouvel analogue de l'insuline basale plus performant, ajout d'une ou plusieurs insulines à action rapide, passage à des insulines prémixées), addition d'un inhibiteur de la dipeptidyl peptidase-4 (gliptine), ajout d'un inhibiteur des cotransporteurs sodium-glucose de type 2 (gliflozine) ou encore la combinaison d'un agoniste des récepteurs du glucagon-like peptide-1 avec I'insuline basale. Le choix doit se faire en fonction du profil et des préférences du patient, dans une approche personnalisée, en tenant compte des avantages et inconvénients de chaque alternative thérapeutique.
\end{abstract}

Therapeutic options for a type 2 diabetic patient not well controlled with metformin plus basal insulin

In a patient with type 2 diabetes not well controlled with a basal insulin - metformin combination, several therapeutic options may be considered : intensifying insulin therapy with different schemes (appropriate titration using a more favourable basal insulin analogue, adding one, two or three rapid-acting insulin analogues, shift to two or three premix insulin injections), adding a dipeptidyl peptidase-4 inhibitor (gliptin) or an inhibitor of sodium-glucose cotransporters type 2 (gliflozin), or combining a glucagon-like peptide-1 receptor agonist with basal insulin. The choice should be made according the individual profile and preference of the patient, in a personalized approach, taking into account the advantages and disadvantages of each therapeutic solution.

\section{INTRODUCTION}

Le diabète de type 2 (DT2) est une maladie complexe, comportant diverses anomalies physiopathologiques, et par ailleurs évolutive dans le temps. Outre la présence d'une insulinorésistance, il se caractérise par un épuisement progressif de la fonction insulinosécrétoire pancréatique. ${ }^{1}$ Il en résulte que la prise en charge thérapeutique nécessite, généralement, une intensification progressive du traitement pharmacologique, en plus des mesures hygiéno-diététiques. Cette stratégie a été résumée dans un article précédent, publié en $2015,{ }^{2}$ rapportant la prise de position commune de l'American Diabetes Association (ADA) et de l'European Association for the Study of

a Université de Liège, Service de diabétologie, nutrition et maladies métaboliques, b Unité de pharmacologie clinique, CHU Sart Tilman, 4000 Liège, Belgique andre.scheen@chu.ulg.ac.be
Diabetes (EASD). ${ }^{3}$ Dans de nombreux cas, l'épuisement de la fonction insulinosécrétoire va aboutir, tôt ou tard, à la nécessité d'instaurer une insulinothérapie. Selon les recommandations précédemment citées, ${ }^{2,3}$ il est proposé de débuter par la prescription d'une insuline basale, avec, le plus souvent et sauf contre-indication, le maintien de la metformine. En effet, d'après une méta-analyse des études comparatives disponibles, la combinaison insuline plus metformine, par comparaison à l'insuline seule, permet d'abaisser le taux d'hémoglobine glyquée ( $\mathrm{HbAlc}$ ) de $0,5 \%$, de diminuer le poids corporel de $1 \mathrm{~kg}$ et de réduire les doses d'insuline de $5 \mathrm{U} /$ jour. $^{4}$ Cependant, ce traitement peut se révéler insuffisant et de nombreux patients n'atteignent pas les objectifs glycémiques sous insuline basale $( \pm$ metformine $) .{ }^{5}$ Se pose alors la question de savoir comment intensifier le traitement antihyperglycémiant, en essayant d'allier efficacité, sécurité, facilité et coût, le tout dans une approche personnalisée centrée sur le patient. . $3,6,6,7^{-}$

Cet article a pour but de discuter les différentes options pharmacologiques qui s'offrent au praticien pour améliorer le contrôle glycémique d'un patient DT2 déjà traité par insuline basale et metformine, mais restant insuffisamment équilibré ${ }^{8,9}$ Les avantages et désavantages des différentes approches ont été analysés en faisant référence à un cas clinique précis dans une contribution récente. ${ }^{10}$ Nous ne discuterons pas ici les éventuelles options consistant à ajouter un sulfamide, un inhibiteur des alpha-glucosidases ou une glitazone, alternatives qui ne sont généralement pas prioritaires, soit pour un manque d'efficacité, soit pour un problème de sécurité d'emploi, ${ }^{11}$ comme souligné dans les dernières recommandations américaines. ${ }^{6,7}$

\section{TITRER L'INSULINE BASALE ET CHOISIR LE MEILLEUR PROFIL PHARMACOCINÉTIQUE}

Il convient, d'abord, de vérifier qu'une titration adéquate a bien été réalisée visant à contrôler la glycémie à jeun selon le concept «treat-to-target». ${ }^{12}$ Différents schémas de titration ont été validés, soit réalisés à intervalles réguliers par l'équipe soignante, soit selon un algorithme décisionnel préétabli qui peut être implémenté par le patient lui-même. Cependant, dans les études cliniques ${ }^{13}$ et sans doute davantage encore dans la pratique courante, ${ }^{5}$ la dose d'insuline basale administrée au coucher est souvent insuffisante et ne permet pas de contrôler la glycémie à jeun de façon optimale.

Ensuite, si le patient est toujours traité par de l'insuline NPH (Neutral Protamine Hagedorn), il convient d'envisager son 


\begin{tabular}{|c|c|c|}
\hline TABLEAU 1 & \multicolumn{2}{|c|}{$\begin{array}{l}\text { Différentes formulations d'insuline } \\
\text { assurant une couverture basale }\end{array}$} \\
\hline \multicolumn{3}{|c|}{ NPH: Neutral Protamine Hagedorn. } \\
\hline Types d'insuline & Nom commercial & Durée d'action \\
\hline $\mathrm{NPH}$ & $\begin{array}{l}\text { Insulatard, Insuman, } \\
\text { (H)Umuline NPH }\end{array}$ & $<24 \mathrm{~h}$ \\
\hline Détémir & Levemir & $<24 \mathrm{~h}$ \\
\hline Glargine U100 & Lantus, Abasaglar & Environ $24 \mathrm{~h}$ \\
\hline Glargine U300 & Toujeo & $>24 \mathrm{~h}$ \\
\hline Dégludec & Tresiba & $>24 \mathrm{~h}$ \\
\hline
\end{tabular}

ductible, couvre mieux les 24 heures, avec un moindre risque hypoglycémique démontré, en particulier durant la période nocturne, et la différence est encore un peu plus marquée avec l'insuline Toujeo. ${ }^{15,16}$ Une autre insuline avec un profil pharmacocinétique favorable est l'insuline dégludec (Tresiba): sa durée d'action dépasse 24 heures, avec un profil plat et reproductible, associé à un plus faible risque d'hypoglycémies nocturnes, certainement que l'insuline NPH et même que l'insuline glargine U100..$^{15,16}$

\section{AJOUTER UNE COMPOSANTE D'INSULINE RAPIDE}

remplacement par un analogue de l'insuline doté d'un profil pharmacocinétique plus adapté (tableau 1) ${ }^{14,15}$ En effet, l'insuline NPH, injectée au coucher, est susceptible d'avoir une action trop importante au milieu de la nuit, exposant ainsi à des hypoglycémies nocturnes, ce qui empêche une titration adéquate permettant de bien contrôler la glycémie du lendemain ; de plus, elle ne permet pas de couvrir correctement la fin de nuit, exposant le sujet à une hyperglycémie au lever, a fortiori s'il existe un phénomène de l'aube (élévation de la glycémie en fin de nuit) ou de Somogyi (rebond post-hypoglycémie)..$^{14,15}$ Des insulines basales avec un meilleur profil pharmacocinétique ont été commercialisées. L’insuline détémir (Levemir) a un profil un peu plus plat, plus long et plus reproductible que l'insuline NPH, mais reste trop courte pour couvrir correctement les 24 heures chez de nombreux patients DT2. ${ }^{14,15}$ Le leader du marché des insulines basales est, actuellement, l'insuline glargine. ${ }^{16}$ Cette insuline a d'abord été commercialisée sous la forme de préparations à $100 \mathrm{U} / \mathrm{ml}$ (Lantus), et cette formulation est maintenant disponible sous la forme d'un biosimilaire (Abasaglar). Plus récemment, l'insuline glargine a été mise à la disposition des médecins dans une préparation plus concentrée, à $300 \mathrm{U} / \mathrm{ml}$ (Toujeo), dotée de propriétés pharmacocinétiques encore un peu plus favorables. ${ }^{14,15}$ D'une façon générale, l'insuline glargine, par rapport à l'insuline NPH, a un profil d'action plus plat et plus repro-

Si le DT2 reste insuffisamment équilibré, malgré ces mesures ciblant spécifiquement l'insuline basale, il est probable que le patient est exposé à des phases d'hyperglycémie postprandiale, non maîtrisées par la seule insuline basale. ${ }^{17} \mathrm{Il}$ convient alors d'envisager l'ajout d'une insuline à action rapide (ou, mieux encore, d'un analogue de l'insuline à action ultrarapide), appelée à neutraliser l'apport glucidique en rapport avec les repas. Il a été proposé de commencer par l'ajout d'un seul bolus d'insuline rapide avant le repas le plus hyperglycémiant de la journée (influence du rythme circadien, mais variable d'un patient à l'autre en fonction des habitudes alimentaires): cette approche est appelée le schéma «basal-plus». Si cette approche s'avère insuffisante, il faut alors ajouter un deuxième, voire un troisième bolus d'insuline à action rapide: l'évolution se fait alors vers un schéma dit «basal-bolus», comme il est couramment pratiqué chez le patient diabétique de type $1 .{ }^{18}$ Les avantages et désavantages de cette approche thérapeutique, en comparaison à celles d'autres alternatives décrites plus loin, sont résumés dans les tableaux 2 et 3, respectivement.

Une autre approche est de remplacer l'insuline basale par deux injections d'insuline prémixée (comportant une proportion, de 25 à $50 \%$ le plus souvent, d'insuline à action rapide),

\begin{tabular}{|c|c|c|c|}
\hline TABLEAU 2 & \multicolumn{2}{|c|}{ Avantages d'une intensification thérapeutique } & \\
\hline \multicolumn{4}{|c|}{$\begin{array}{l}\text { Comparaison des avantages des quatre approches d'intensification thérapeutique après échec d'un traitement par insuline basale ( } \pm \text { metformine). } \\
\text { DPP-4i: inhibiteur de la dipeptidyl peptidase-4. SGLT2i: inhibiteur des cotransporteurs sodium-glucose de type } 2 \text {. AR GLP-1: agoniste des récepteurs du glucagon-like } \\
\text { peptide-1. }\end{array}$} \\
\hline Ajout d'une insuline rapide & Ajout d'un DPP-4i & Ajout d'un SGLT2i & Ajout d'un AR GLP-1 \\
\hline $\begin{array}{l}\text { - La plus flexible } \\
\text { - En principe la plus efficace (titration } \\
\text { jusqu’à l'objectif) } \\
\text { - Pas de contre-indication } \\
\text { si insuffisance rénale }\end{array}$ & $\begin{array}{l}\text { - Prise orale (simplicité) } \\
\text { - Pas de prise de poids } \\
\text { - Pas d’hypoglycémie } \\
\text { - Excellente tolérance } \\
\text { - Sécurité cardiovasculaire } \\
\text { - Pas de contre-indication } \\
\text { si insuffisance rénale }\end{array}$ & $\begin{array}{l}\text { - Prise orale (simplicité) } \\
\text { - Perte de poids } \\
\text { - Pas d'hypoglycémie } \\
\text { - Réduction des besoins insuliniques } \\
\text { - Protection cardiovasculaire } \\
\text { (empagliflozine) } \\
\text { - Option à privilégier si insuffisance } \\
\text { cardiaque }\end{array}$ & $\begin{array}{l}\text { - Peu de contrainte } \\
\text { (surtout si injection hebdomadaire) } \\
\text { - Perte de poids } \\
\text { - Pas d’hypoglycémie } \\
\text { - Réduction des besoins insuliniques } \\
\text { - Protection cardiovasculaire } \\
\quad \text { (liraglutide) }\end{array}$ \\
\hline
\end{tabular}

TABLEAU 3

Désavantages d'une intensification thérapeutique

Comparaison des désavantages des quatre approches d'intensification thérapeutique après échec d'un traitement par insuline basale ( \pm metformine). DPP-4i: inhibiteur de la dipeptidyl peptidase-4. SGLT2i: inhibiteur des cotransporteurs sodium-glucose de type 2. AR GLP-1: Agoniste des récepteurs du glucagon-like peptide-1.

\begin{tabular}{|c|c|c|c|}
\hline Ajout d'une insuline rapide & Ajout d'un DPP-4i & Ajout d'un SGLT2i & Ajout d'un AR GLP-1 \\
\hline $\begin{array}{l}\text { - Injection(s) quotidiennes(s) } \\
\text { - Titration (plus contraignant) } \\
\text { - Autosurveillance glycémique } \\
\text { - Besoin d'une éducation thérapeutique } \\
\text { - Risque d’hypoglycémie } \\
\text { - Risque de prise de poids }\end{array}$ & $\begin{array}{l}\text { - Un peu moins puissant } \\
\text { (surtout si HbA1c élevée) } \\
\text { - Pas de protection cardiovasculaire } \\
\text { démontrée }\end{array}$ & $\begin{array}{l}\text { - Infections génitales } \\
\text { - Contre-indication si insuffisance } \\
\text { rénale }\end{array}$ & $\begin{array}{l}\text { - Troubles digestifs (transitoires) } \\
\text { - Coût plus élevé }\end{array}$ \\
\hline
\end{tabular}




\begin{tabular}{|c|c|c|c|c|}
\hline & TABLEAU 4 & \multicolumn{2}{|c|}{$\begin{array}{c}\text { Comparaison de différentes méta-analyses des essais } \\
\text { cliniques contrôlés }\end{array}$} & \\
\hline \multirow{2}{*}{\multicolumn{5}{|c|}{$\begin{array}{l}\text { Ce tableau compare les résultats de différentes méta-analyses des essais cliniques contrôlés dans lesquels une intervention pharmacologique (autre que } \\
\text { l'intensification de l'insulinothérapie en elle-même) complète le traitement par insuline basale. Les résultats correspondent aux différences moyennes pondérées } \\
\text { (avec intervalle de confiance à } 95 \%) \text {. } \\
\text { (*) ND: non disponible }\left(-2,59 \mathrm{UI} / \text { jour, p }<0,0001 \text { versus placebo, dans la méta-analyse de Charbonnel et coll. }{ }^{34} \text { consacrée au lixisénatide). }\right. \\
\text { DPP-4i: inhibiteur de la dipeptidyl peptidase-4. SGLT2i: inhibiteur des cotransporteurs sodium-glucose de type } 2 \text {. AR GLP-1: Agoniste des récepteurs du glucagon-like } \\
\text { peptide-1. }\end{array}$}} \\
\hline & & & & \\
\hline \multicolumn{2}{|c|}{ Critères d'évaluation } & Ajout d'un DPP-4i & Ajout d'un SGLT2i & Ajout d'un AR GLP-1 \\
\hline \multicolumn{2}{|l|}{ Référence } & Kim et coll., $2016^{22}$ & Tang et coll., $2017^{29}$ & Wysham et coll., $2017^{33}$ \\
\hline \multicolumn{2}{|c|}{$\mathrm{N}$ essais/n patients } & $9 / 4464$ & $7 / 5801$ & $19 / 7053$ \\
\hline \multicolumn{2}{|c|}{$\mathrm{HbA1c}(\%)$} & $-0,58(-0,70 ;-0,46)$ & $-0,56(-0,67 ;-0,44)$ & $-0,48(-0,67 ;-0,30)$ \\
\hline \multicolumn{2}{|c|}{ Glycémie à jeun (mmol/l) } & $-0,59(-0,79 ;-0,40)$ & $-0,95(-1,21 ;-0,70)$ & $-0,27(-0,49 ;-0,05)$ \\
\hline \multicolumn{2}{|c|}{ Glycémie post-prandiale (mmol/l) } & ND & ND & $-2,61(-3,72 ;-1,51)$ \\
\hline \multicolumn{2}{|c|}{ Dose d'insuline (UI/jour) } & $-1,86(-3,27 ;-0,45)$ & $-8,79(-13,4 ;-0,22)$ & $\mathrm{ND}(*)$ \\
\hline \multicolumn{2}{|c|}{ Poids corporel $(\mathrm{kg})$} & $-0,04(-0,25 ; 0,16)$ & $-2,63(-3,10 ;-0,16)$ & $-2,60(-3,32 ;-1,89)$ \\
\hline \multicolumn{2}{|c|}{ Risque hypoglycémique (odds ratio) } & $0,94(0,84 ; 1,05)$ & $1,07(0,99 ; 1,15)$ & $0,92(0,68 ; 1,23)$ \\
\hline
\end{tabular}

généralement avant le repas du matin et du soir. Cette solution a cependant le désavantage d'être moins flexible que le schéma basal-plus ou basal-bolus pour ce qui concerne l'ajustement précis des doses d'insuline à action lente et rapide en fonction du profil glycémique du patient. ${ }^{2,3}$ De plus, elle a été associée à un risque accru d'hypoglycémie et de prise de poids. Cependant, l'analyse comparative des deux schémas insuliniques donne des résultats différents selon les études publiées et, au final, les deux approches apparaissent sensiblement équivalentes..$^{19,20} \mathrm{Il}$ est d'ailleurs intéressant de noter que le schéma basal-plus/bolus était privilégié dans la prise de position ADA/EASD de $2015^{3}$ alors que le schéma insulinique faisant appel aux insulines prémixées est maintenant placé sur un pied équivalent dans l'algorithme proposé dans le «Standards of medical care in diabetes» de l'ADA en 2017 . $^{7}$ Le choix peut donc se faire en fonction du profil et de la préférence du patient.

\section{AJOUTER UN INHIBITEUR DE LA DPP-4}

Les inhibiteurs de la dipeptidyl peptidase-4 (DPP-4), mieux connus sous le nom de gliptines, ${ }^{21}$ ouvrent de nouvelles possibilités thérapeutiques, et ce également en ajout à une insuline basale. ${ }^{22}$ Par rapport à un sulfamide, les gliptines offrent l'avantage d'un risque hypoglycémique nettement diminué, d'une neutralité pondérale et d'une plus grande facilité d'utilisation (une prise par jour et pas de titration à l'instauration du traitement). Ces mêmes avantages se retrouvent aussi en comparaison avec l'ajout d'une insuline prandiale (tableau 2). De plus, les inhibiteurs de la DPP-4 peuvent être utilisés de façon sûre chez les patients avec une insuffisance rénale, contrairement à la plupart des sulfamides. Ils ont, par ailleurs, démontré leur sécurité cardiovasculaire dans plusieurs grands essais cliniques prospectifs, y compris chez des patients DT2 déjà traités par insuline. ${ }^{23}$ La réduction du taux d'HbAlc escomptée avec l'ajout d'une gliptine à une insuline basale (metformine) est cependant relativement limitée, de l'ordre de $0,5 \%$ en moyenne, et moins importante que lorsque l'inhibiteur de la DPP-4 est ajouté à la metformine (en général $0,7-0,8 \%) .{ }^{21}$ L'ajout d'une gliptine à une insuline basale ( \pm metformine) peut donc être envisagé uniquement si le taux d'HbAic n'est pas trop éloigné de l'objectif fixé pour le patient, ce qui représente une limitation pour ce qui concerne cette solution thérapeutique (tableau 3). Par contre, l'ajout d'une gliptine à une insuline basale améliore le contrôle glycémique avec une dose plus faible d'insuline, sans faire prendre du poids et sans aggraver le risque hypoglycémique, comme cela a été résumé dans une méta-analyse récente (tableau 4). ${ }^{22}$ De plus, la gliptine a un excellent profil de tolérance, y compris chez le sujet âgé plus fragile et chez le patient avec une insuffisance rénale (tableau 2). Il s'agit donc certainement là d'une option intéressante dans ces populations à risque. $^{24}$

\section{AJOUTER UN INHIBITEUR DES SGLT2}

La commercialisation, plus récente, des inhibiteurs des cotransporteurs sodium-glucose de type 2 (SGLT2) rénaux, appelés aussi gliflozines, offre une autre alternative thérapeutique, y compris chez des patients DT2 déjà traités par insuline. ${ }^{25}$ Ce type de médicament, en favorisant une glucosurie, améliore le contrôle glycémique tout en faisant perdre du poids. De plus, l'effet natriurétique concomitant s'accompagne d'une baisse de la pression artérielle et un effet hypouricémiant a également été démontré. ${ }^{25}$ Par ailleurs, les résultats de l'étude EMPA-REG OUTCOME avec l'empagliflozine, démontrant une protection cardiovasculaire et rénale, ouvrent de nouvelles perspectives chez les patients à haut risque cardiovasculaire. ${ }^{26,27}$ C'est d'autant plus intéressant que les patients DT2 requérant une insulinothérapie basale ont, le plus souvent, une longue durée de diabète avec, potentiellement, une atteinte cardiovasculaire et/ou rénale au moins débutante. Dans l'étude EMPA-REG OUTCOME, 48\% des patients DT2 bénéficiaient d'une insulinothérapie, sans différence significative dans les critères d'évaluation entre les patients traités ou non par insuline, mais le pourcentage de patients uniquement sous insuline basale n'est pas renseigné. ${ }^{26}$ Ainsi, les inhibiteurs SGLT2 peuvent dorénavant être proposés comme une alternative intéressante aux inhibiteurs de la DPP-4, au moins chez les patients avec un profil de haut risque cardiovasculaire. Rappelons cependant que les gliflozines, contrairement aux gliptines, ne peuvent être prescrites actuellement chez les patients DT2 avec un débit de filtration glomérulaire estimé en-dessous de $60 \mathrm{ml} / \mathrm{min} / 1,73 \mathrm{~m}^{2}$. Un 
intérêt particulier peut être trouvé chez le patient DT2 avec une insuffisance cardiaque, comme souligné dans les dernières recommandations de la Société européenne de cardiologie. ${ }^{28}$ Ce positionnement particulier résulte de l'action diurétique/natriurétique des inhibiteurs des SGLT2 et des résultats de l'étude EMPA-REG OUTCOME montrant une réduction significative de $35 \%$ des hospitalisations pour insuffisance cardiaque avec l'empagliflozine. ${ }^{27}$

En association avec l'insuline, et si l'on compare les résultats de deux méta-analyses, les inhibiteurs des SGLT2 ${ }^{29}$ améliorent le taux d'HbAic de façon assez comparable à ce qui a été rapporté avec les inhibiteurs de la DPP- $4 .{ }^{22}$ Cependant, ils offrent l'avantage d'entraîner une perte de poids et une réduction des doses journalières d'insuline plus importantes que les gliptines, avec un risque hypoglycémique faible et comparable (tableau 4).

Les inhibiteurs des SGLT2 sont, en général, bien tolérés, mais il existe un risque accru d'infections génitales, lié aux mécanismes d'action. La balance bénéfices/risques de cette classe pharmacologique est considérée comme favorable, y compris en combinaison avec l'insuline. ${ }^{30}$

\section{AJOUTER UN AGONISTE DES RÉCEPTEURS DU GLP-1}

La dernière prise de position ADA-EASD ouvre la voie à un traitement combinant une insuline basale et un agoniste des récepteurs du glucagon-like peptide-1 (GLP-1) plutôt que de passer à un schéma insulinique basal-plus, voire basal-bolus, ou à des insulines prémixées. ${ }^{2,3}$ La combinaison d'un agoniste des récepteurs du GLP-1 et d'une insuline basale est de plus en plus mise en avant dans la prise en charge du DT2 à un stade relativement avancé, de façon à cibler à la fois la glycémie postprandiale et la glycémie à jeun..$^{31,32}$ Rappelons que le GLP-1 est une hormone incrétine qui stimule la sécrétion d'insuline, mais aussi réduit la sécrétion de glucagon; ces deux actions combinées se font de façon gluco-dépendante, ce qui limite le risque hypoglycémique. Par ailleurs, les agonistes des récepteurs du GLP-1 réduisent également l'appétit par un effet sur le centre de la satiété (comme avec le liraglutide) ou par un effet périphérique de ralentissement de la vidange gastrique (comme avec l'exénatide ou le lixisénatide). Cette combinaison agoniste des récepteurs du GLP-1-insuline basale réduit significativement le taux d'HbAic par rapport à l'insuline seule, avec une action sur les glycémies à jeun mais surtout postprandiales, tout en faisant perdre du poids et sans aggraver significativement le risque hypoglycémique. Ces données favorables ont été résumées dans une méta-analyse récente regroupant les résultats obtenus avec tous les agonistes des récepteurs du GLP-1 (tableau 4), ${ }^{33}$ confirmant ceux d'une autre méta-analyse consacrée au seul lixisénatide. ${ }^{34} \mathrm{Par}$ ailleurs, cette combinaison a démontré divers avantages par rapport à un schéma insulinique basal-bolus avec, dans les différentes études comparatives disponibles, une meilleure réduction du taux d'HbAlc, une diminution notable des besoins en insuline, un moindre risque d'hypoglycémies et une perte de poids, contrastant avec la prise pondérale généralement observée avec l'intensification par un schéma insulinique basal-bolus. ${ }^{35-37}$ Cette combinaison thérapeutique, certes plus onéreuse si l'on s'en tient strictement aux prix comparatifs des médicaments, nécessite moins de mesures d'autosurveillance glycémique qu'un schéma basal-bolus et l'éducation thérapeutique est, en général, facilitée.

Dans l'étude LEADER, le liraglutide a réduit significativement le critère cardiovasculaire composite primaire de $13 \%$, la mortalité cardiovasculaire de $22 \%$ et la mortalité de toutes causes de $15 \%$ chez des patients DT2 dont l'immense majorité étaient en prévention secondaire. ${ }^{38}$ Ces résultats ont été commentés dans un article précédent de la revue. ${ }^{27}$ Dans cette étude, plus d'un tiers des patients bénéficiaient d'une insulinothérapie basale à l'entrée dans l'étude (22,3\% sous insuline longue et $11,7 \%$ sous insuline intermédiaire) et, dans le décours du suivi, respectivement $13,3 \%$ et $5,8 \%$ de patients supplémentaires ont reçu ces deux types d'insuline basale dans le bras liraglutide (versus $20,1 \%$ et $8,3 \%$, respectivement, dans le bras placebo).$^{38} \mathrm{La}$ combinaison liraglutide-insuline basale peut donc être recommandée chez les patients DT2 à haut risque cardiovasculaire, en particulier en prévention secondaire, comme cela est maintenant reconnu dans la dernière version des modalités de traitement du DT2 par l'American Diabetes Association. $^{?}$

Plusieurs agonistes des récepteurs du GLP-1 sont disponibles, soit en injection quotidienne (exénatide (Byetta), lixisénatide (Lyxumia), liraglutide (Victoza)), soit en injection hebdomadaire (exénatide à libération retardée (Bydureon), albiglutide (Eperzan), dulaglutide (Trulicity)). La commercialisation de formulations à injection hebdomadaire pourrait favoriser le recours à cette approche thérapeutique à l'avenir, essentiellement pour des raisons de commodités pour le patient, mais elles n'ont pas encore montré de bénéfices sur le plan cardiovasculaire (études en cours). ${ }^{39}$ Enfin, signalons que diverses combinaisons fixes «insuline basale-agoniste des récepteurs du GLP-1» ont été admises sur le marché pour le traitement du DT2 par l'Agence européenne du médicament en une injection par jour : il s'agit de l'IdegLira (combinaison fixe d'insuline dégludec et de liraglutide, Xultophy) et du LixiLan (combinaison fixe d'insuline glargine et de lixisénatide, Suliqua) ${ }^{32,40}$ Une méta-analyse récente a montré que le passage d'une insuline basale à une combinaison fixe de ce type est associé à une réduction de $0,72 \%$ du taux d'HbAic (intervalle de confiance à $95 \%$ : $-1,03$ à $-0,41 \%$ ), à une perte pondérale de $2,35 \mathrm{~kg}(-3,52$ à $-1,19 \mathrm{~kg})$ et à une diminution du risque d'hypoglycémie (odds ratio: 0,70; 0,57 à 0,86), mais au prix d'une incidence accrue de nausées, surtout en début de traitement (odds ratio: 6,89; 3,73-12,74).40

\section{CONCLUSION}

Le médecin dispose maintenant de plusieurs options thérapeutiques pour optimiser un traitement par insuline basale associé à la metformine. Une intensification du traitement doit être envisagée si l'objectif glycémique en termes d'HbAic n'est pas atteint, en insistant sur le fait que cet objectif doit être individualisé en fonction du profil clinique de chaque patient DT2. Chaque solution discutée dans cet article offre des avantages, mais est aussi potentiellement grevée d'inconvénients. Il convient donc d'informer le patient de la balance avantages/désavantages de façon à choisir, de concert avec lui, la meilleure option thérapeutique. Il est évident que, 
quelle que soit la solution choisie, un suivi clinique et biologique régulier s'impose de façon à pouvoir changer d'option si les résultats ne sont pas jugés satisfaisants en termes d'efficacité ou de tolérance et ce, d'autant plus que la maladie DT2 continuera à évoluer avec un épuisement progressif de la fonction insulinosécrétoire des cellules bêta. Il est possible qu'à terme, plusieurs des options décrites dans cet article puissent être combinées pour encore améliorer l'efficacité, mais une validation préalable basée sur les principes de la médecine factuelle s'impose.

Conflits d'intérêts: A. Scheen déclare avoir reçu des honoraires comme orateur, membre d'un conseil scientifique ou investigateur clinicien de la part des firmes AstraZeneca, Boehringer Ingelheim, Janssen, Lilly, Merck Sharp \& Dohme, Novartis, NovoNordisk, et Sanofi. Il a été investigateur clinicien dans les études EMPA-REG OUTCOME, CANVAS-R, TECOS et LEADER.

N. Paquot a reçu des honoraires comme orateur de la part des firmes Merck Sharp \& Dohme et NovoNordisk.

1 DeFronzo RA. Banting Lecture. From the triumvirate to the ominous octet: $A$ new paradigm for the treatment of type 2 diabetes mellitus. Diabetes 2009;58:773-95. 2 *Scheen AJ, Paquot N. Actualisation 2015 du traitement de l'hyperglycémie dans le diabète de type 2 . Rev Med Suisse 2015;11:1518-25.

3 Inzucchi SE, Bergenstal RM, Buse JB, et al. Management of hyperglycaemia in type 2 diabetes, 2015: A patient-centred approach. Update to a position statement of the American Diabetes Association and the European Association for the Study of Diabetes. Diabetologia 2015;58:429-42. 4 Hemmingsen B, Christensen LL, Wetterslev J, et al. Comparison of metformin and insulin versus insulin alone for type 2 diabetes: Systematic review of randomised clinical trials with meta-analyses and trial sequential analyses. BMJ 2012; 344:e1771. 5 Dalal MR, Grabner M, Bonine N, et al. Are patients on basal insulin attaining glycemic targets? Characteristics and goal achievement of patients with type 2 diabetes mellitus treated with basa insulin and physician-perceived barriers to achieving glycemic targets. Diabetes Res Clin Pract 2016;121:17-26.

6 Garber AJ, Abrahamson MJ, Barzilay $\mathrm{Jl}$, et al. Consensus statement by the American Association of Clinical Endocrinologists and American College of Endocrinology on the comprehensive type 2 diabetes management algorithm 2017 executive summary. Endocr Pract 2017;23:207-238.

7 American Diabetes Association. Standards of medical care in diabetes 2017. Diabetes Care 2017:40:S1-S135. 8 Meneghini LF. Intensifying insulin therapy: what options are available to patients with type 2 diabetes? Am J Med 2013;126:S28-37.

9 Raccah D. Basal insulin treatment intensification in patients with type 2 diabetes mellitus: A comprehensive systematic review of current options. Diabetes Metab 2017;43:110-24. 10 *Scheen AJ, Paquot N. Optimisation d'un traitement par insuline basale chez le patient diabétique de type 2 . Rev Med Liege 2017;72:156-61.

$11 *$ Vos RC, van Avendonk MJ, Jansen H, et al. Insulin monotherapy compared with the addition of oral glucose-lowering agents to insulin for people with type 2 diabetes already on insulin therapy and inadequate glycaemic control. Cochrane Database Syst Rev 2016; 9:CD006992. 12 Strange P. Treat-to-target insulin titration algorithms when initiating long or intermediate acting insulin in type 2 diabetes. J Diabetes Sci Technol 2007;1:540-8.

13 Scheen AJ, Schmitt H, Jiang HH, et al. Factors associated with reaching or not reaching target $\mathrm{HbA1c}$ after initiation of basal or premixed insulin in patients with type 2 diabetes. Diabetes Metab 2017;43:69-78.

14 Pettus J, Santos Cavaiola T, Tamborlane WV, et al. The past, present, and future of basal insulins. Diabetes Metab Res Rev 2016;32:478-96.

15 Goldman J, Kapitza C, Pettus J, et al. Understanding how pharmacokinetic and pharmacodynamic differences of basal analog insulins influence clinical practice. Curr Med Res Opin 2017;1-26.

16 Woo VC. A review of the clinical efficacy and safety of insulin degludec and glargine $300 \mathrm{U} / \mathrm{mL}$ in the treatment of diabetes mellitus. Clin Ther 2017; epub ahead of print.

17 Raccah D, Chou E, Colagiuri S, et al. A global study of the unmet need for glycemic control and predictor factors among patients with type 2 diabetes mellitus who have achieved optimal fasting plasma glucose control on basa insulin. Diabetes Metab Res Rev 2017;33. 18 Owens DR. Stepwise intensification of insulin therapy in type 2 diabetes management--exploring the concept of the basal-plus approach in clinical practice. Diabet Med 2013;30:276-88. 19 Scheen AJ, Schmitt H, Jiang HH, et al. Individualizing treatment of type 2 diabetes by targeting postprandial or fasting hyperglycaemia: Response to a basal vs a premixed insulin regimen by $\mathrm{HbA1c}$ quartiles and ethnicity. Diabetes Metab 2015;41:216-22.

20 Downie M, Kilov G, Wong J. Initiation and intensification strategies in type 2 diabetes management: A comparison of basal plus and premix regimens. Diabetes Ther 2016;7:641-57.

21 Scheen AJ. A review of gliptins for 2014. Exp Opin Pharmacother 2015;16:43-62.

\section{IMPLICATIONS PRATIOUES}

- Un analogue d'insuline basale doté d'une meilleure pharmacocinétique facilite une titration optimale, à compléter éventuellement par l'ajout d'une insuline à action (ultra-) rapide

- La solution la plus aisée, mais pas nécessairement la plus efficace, consiste à ajouter à la combinaison insuline basalemetformine un autre antidiabétique oral, soit une gliptine, soit une gliflozine

- L'ajout d'un agoniste des récepteurs du GLP-1 à une insuline basale ( \pm metformine) est une solution simple, efficace et sûre, mais plus onéreuse

- Ces différentes options, permettant une approche personnalisée, devraient éviter l'inertie clinique trop fréquemment rencontrée dans la prise en charge d'un diabète de type 2
22 Kim YG, Min SH, Hahn S, et al. Efficacy and safety of the addition of a dipeptidy peptidase-4 inhibitor to insulin therapy in patients with type 2 diabetes: A systematic review and meta-analysis. Diabetes Res Clin Pract 2016;116:86-95. 23 Scheen AJ. Gliptines, sécurité cardio-vasculaire et insuffisance cardiaque: le point après l'étude TECOS. Rev Med Suisse 2015;11:1526-31. 24 Scheen AJ. Safety of dipeptidyl peptidase- 4 inhibitors for treating type 2 diabetes. Expert Opin Drug Saf 2015;14:505-24.

25 Scheen AJ. Pharmacodynamics, efficacy and safety of sodium-glucose co-transporter type 2 (SGLT2) inhibitors for the treatment of type 2 diabetes mellitus. Drugs 2015;75:33-59.

26 Zinman B, Wanner C, Lachin JM, et al. Empagliflozin, cardiovascular outcomes, and mortality in type 2 diabetes. $N$ Engl Med 2015;373:2117-28.

27 Scheen AJ, Wallemacq C, Jandrain B, et al. Protection cardiovasculaire du patient diabétique de type 2: d'EMPA-REG OUTCOME à LEADER. Rev Med Suisse 2016;12:1370-5.

28 Ponikowski P, Voors AA, Anker SD, et al. 2016 ESC Guidelines for the diagnosis and treatment of acute and chronic heart failure: The Task Force for the diagnosis and treatment of acute and chronic heart failure of the European Society of Cardiology (ESC)Developed with the special contribution of the Heart Failure Association (HFA) of the ESC. Eur Heart J 2016:37:2129-200.

29 Tang H, Cui W, Li D, et al. Sodium-glucose co-transporter 2 inhibitors in addition to insulin therapy for management of type 2 diabetes mellitus: A meta-analysis of randomized controlled trials. Diabetes Obes Metab

2017;19:142-7.

30 Scheen AJ. SGLT2 inhibitors: Benefit/ risk balance. Curr Diabetes Rep 2016;16:92.

31 Balena R, Hensley IE, Miller S, et al. Combination therapy with GLP-1 receptor agonists and basal insulin: A systematic review of the literature. Diabetes Obes Metab 2013;15:485-502.

32 Owens DR, Monnier L, Barnett AH. Future challenges and therapeutic opportunities in type 2 diabetes:
Changing the paradigm of current therapy. Diabetes Obes Metab 2017; epub ahead of print.

33 Wysham CH, Lin J, Kuritzky L. Safety and efficacy of a glucagon-like peptide-1 receptor agonist added to basal insulin therapy versus basal insulin with or without a rapid-acting insulin in patients with type 2 diabetes: Results of a meta-analysis. Postgrad Med 2017;129:436-45.

34 Charbonnel B, Bertolini M, Tinahones $\mathrm{FJ}$, et al. Lixisenatide plus basal insulin in patients with type 2 diabetes mellitus: A meta-analysis. J Diabetes Complications 2014;28:880-6.

35 *Scheen AJ, Paquot N. Intérêt d'une combinaison agoniste des récepteurs du GLP-1 et insuline basale dans le traitement du diabète de type 2 . Rev Med Suisse 2014;10:1549-54.

36 Giorgino F, Bonadonna RC, Gentile S, et al. Treatment intensification in patients with inadequate glycemic control on basal insulin: rationale and clinical evidence for the use of short-acting and other glucagon-like peptide- 1 receptor agonists. Diabetes Metab Res Rev 2016;32:497-511.

37 Rosenstock J, Guerci B, Hanefeld M, et al. Prandial options to advance basal insulin glargine therapy: Testing lixisenatide plus basal insulin versus insulin glulisine either as basal-plus or basal-bolus in type 2 diabetes: the GetGoal Duo-2 trial. Diabetes Care 2016;39:1318-28.

38 Marso SP, Daniels GH, Brown-Frandsen $\mathrm{K}$, et al. Liraglutide and cardiovascular outcomes in type 2 diabetes. $N$ Engl J Med 2016;375:311-22.

39 Scheen AJ. Historique des études cardiovasculaires: de l'UGDP ... aux dernières études. Médecine des maladies métaboliques 2017;11:2S15-2S26. 40 Liakopoulou P, Liakos A, Vasilakou D, et al. Fixed ratio combinations of glucagon like peptide 1 receptor agonists with basal insulin: A systematic review and meta-analysis. Endocrine 2017;56:485-94. 